\title{
Asystole Following Nitroglycerin: A Review of Two Cases
}

Brian M. Pennington, MD; Christopher M. Howell, DSc, MSc, MPAS, PA-C, MBA; Ryan Babienco, MD

\section{Case reports of a 54-year-old man with angina and a 69-year-old woman demonstrate an underreported, self-limiting side effect associated with nitroglycerin.}

$\mathrm{N}$ itroglycerin (NTG), or glyceryl trinitrate, was first introduced into the medical community by Murrell, ${ }^{1,2}$ who reported on anecdotal observations of its antianginal properties by workers within manufacturing plants refining the product for its explosive properties. While the route of administration of NTG has changed from this incidental environmental exposure to the now formulated therapies available, its benefit as an outpatient, abortive treatment for stable angina has been validated beyond early subjective observations in the literature ${ }^{1-3}$ In fact, its successful use over the years for angina has produced an expansive pharmacopeia, including its use for undifferentiated chest pain and exacerbation of congestive heart failure..$^{3-5}$

Despite the extensive history of NTG as a proven vasodilator, emerging uses continue to be explored in equal measure with technological advances. ${ }^{2,6}$ Though morbidity and mortality reductions are dependent on its use within clinical practice, NTG is not an innocuous drug. ${ }^{5}$ Most of the reported side effects associated with NTG are well established and include hypotension, tachycardia, flushing, nausea, vomiting, and headache. ${ }^{3,6,7} \mathrm{An}$ often forgotten side effect associated with NTG use is asystole. We present the following two cases to highlight both common uses of NTG as well as this underreported side effect.

\section{Case 1: Nitroglycerin for Stable Anginal Chest Pain}

A 54-year-old man with a history of hypertension (HTN), hyperlipidemia (HLP), and gastroesophageal reflux disease (GERD) presented to the ED for evaluation of a 3-hour history of intermittent, retrosternal, left-sided, nonradiating chest "pressure and tightness." The patient stated that the chest discomfort began at rest but was exacerbated by exertion with episodes lasting 10 to 15 minutes. The patient rated the peak pain associated with these episodes as a "7" on a pain scale of 1 to 10. He further noted that his symptoms abated and he became "pain-free" when at rest.

The patient's vital signs at presentation were: blood pressure (BP), 156/87 mm Hg; heart rate (HR), 68 beats/min; respiratory rate (RR), 18 beats/min; and temperature (T), $98.4^{\circ} \mathrm{F}$. Oxygen saturation was $96 \%$ on room air.

Dr Pennington is an assistant professor of emergency medicine and ultrasound director, Wright State University Boonshoft School of Medicine, Department of Emergency Medicine, Kettering, Ohio. Dr Howell is a physician assistant and associate professor, Kettering College Master of Physician Assistant Studies, Kettering, Ohio. Dr Babienco is an emergency medicine resident, Wright State University Boonshoft School of Medicine, Department of Emergency Medicine, Kettering, Ohio.

Authors' Disclosure Statement: The authors report no actual or potential conflict of interest in relation to this article.

DOI: 10.12788/emed.2018.0102 


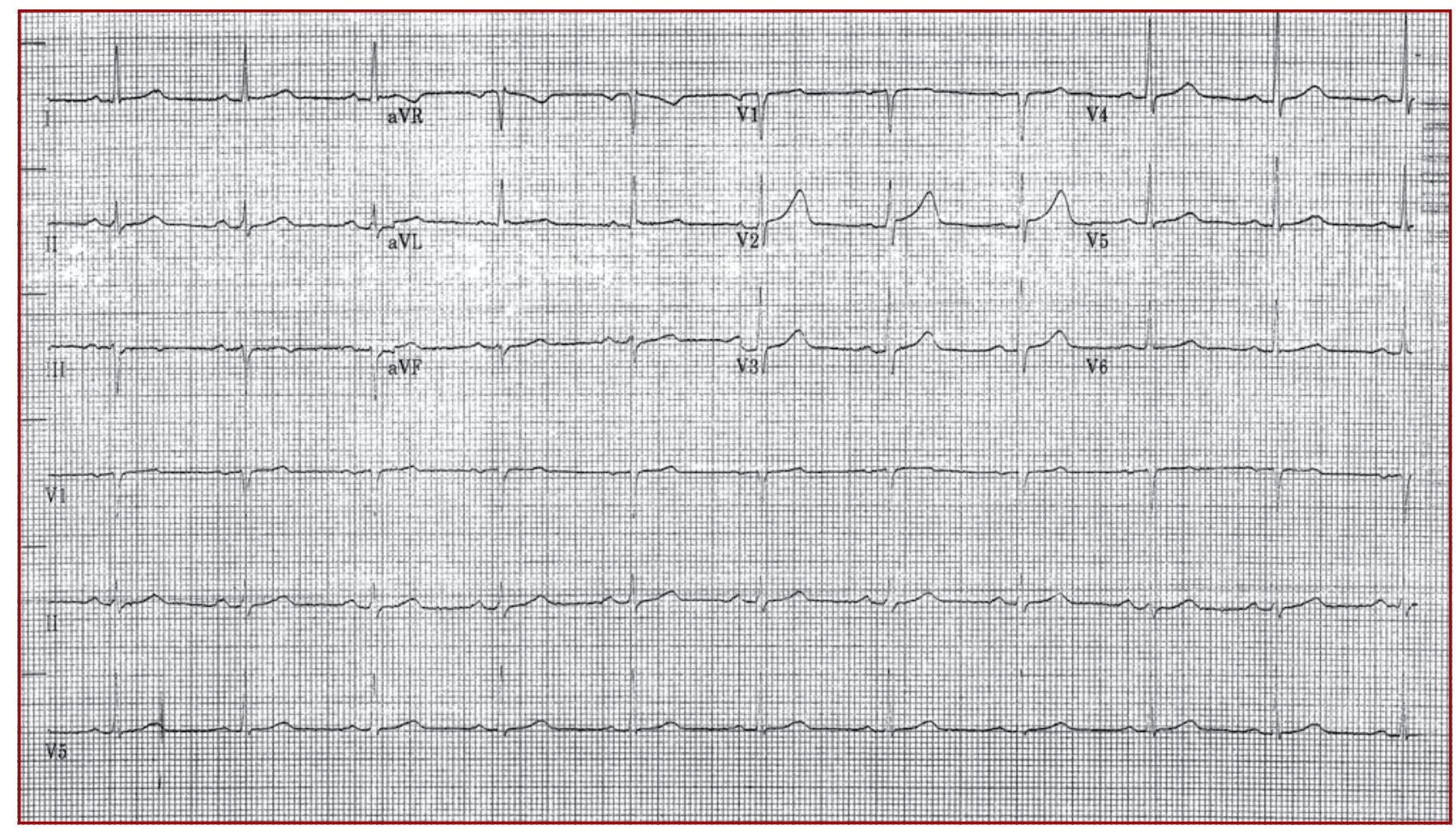

Figure 1. Electrocardiogram of the patient in Case 1 demonstrates a normal sinus rhythm, left axis deviation, normal intervals, and no acute ST-segment elevation or depression.

The patient, who performed regular BP checks at home, noted that his recent BP readings had been very high. A review of the patient's systems was positive for shortness of breath and diaphoresis; symptoms were otherwise negative, including any prior episodes. His social history was noncontributory and negative for tobacco, alcohol, or drug use. The patient did report that he had taken an uneventful 6-hour car ride the previous week.

On physical examination, the patient was nontoxic and resting comfortably, without signs of acute distress or pain. Cardiac and pulmonary examinations were normal, and radial pulses were $2+$ and symmetric. The abdominal examination was benign and the neurological examination was nonfocal. There was no evidence of peripheral edema or asymmetry of the calves, which were nontender to palpation.

The initial electrocardiogram (ECG) (Figure 1) showed a normal sinus rhythm of 65 beats/min, left axis deviation, and normal intervals; there was no acute ST-segment elevation or depression.

\section{Case 2: Nitroglycerin for Unstable Anginal Chest Pain}

A 69-year-old obese woman with a medical history significant for HTN, HLP, and GERD presented to the ED for evaluation of nausea and chest pressure. She described the chest pressure as feeling dull and heavy. She further noted that the discomfort had been occurring intermittently upon exertion, but that this recent episode started while at rest and persisted.

The patient's vital signs at presentation were: BP, 183/80 mm Hg; HR, 94 beats/min; RR, 20 beats/min; and $\mathrm{T}, 98.0^{\circ} \mathrm{F}$. Oxygen saturation was $92 \%$ on room air. On a review of 


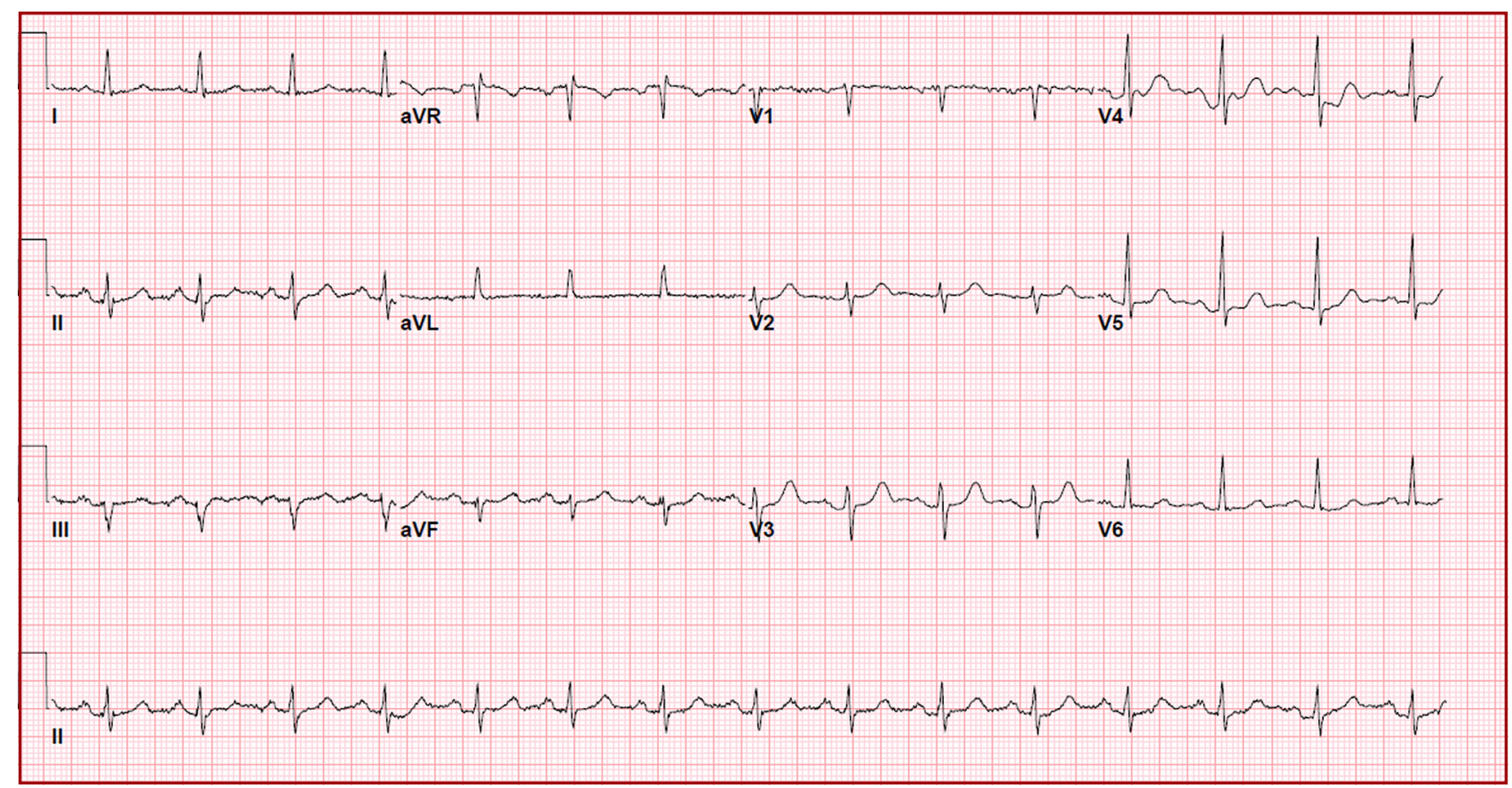

Figure 2. Electrocardiogram taken of the patient in Case 2 demonstrates normal sinus rhythm with no ST-segment changes or T-wave inversions.

systems, the patient denied any associated symptoms; she likewise denied a history of any recent surgeries, immobilization, active malignancy, or recent travel. Her social history was noncontributory and was negative for tobacco, alcohol, or recreational drug use.

On physical examination the patient was nontoxic and resting comfortably, without signs of acute distress or diaphoresis. The cardiac and pulmonary examinations were normal, and radial pulses were $2+$ and symmetric. The patient had trace pedal edema bilaterally, but her calves were symmetric and nontender. The abdomen was benign and the neurological examination was nonfocal. An ECG (Figure 2) showed a normal sinus rhythm with no signs of ischemia (eg, no ST-segment changes or T-wave inversions were present).

\section{Cases 1 and 2: Shared Clinical Course}

In both of the two cases presented, ECGs were obtained for the patients upon arrival at the ED. Both patients were placed on telemetry with continuous monitoring, and intravenous (IV) access was obtained. Baseline laboratory evaluation for each of these patients included a complete blood count, basic metabolic panel, and cardiac enzyme measurement. A D-dimer test was also ordered for the patient in Case 1 based on his concerning history and low-pretest probability for a pulmonary embolism (ie, positive pulmonary embolism rule-out criteria). Portable chest X-ray imaging on each of the patients showed no acute pathology, and all of their laboratory results were within normal ranges. Both of the patients in Case 1 and 2 received a 324-mg chewable aspirin and an IV fluid bolus.

\section{Case 1}

During evaluation, the patient in Case 1 developed unprovoked chest pain, which he rated as a "7," for which he was given 400 mcg NTG sublingually (SL). After administra- 
tion of NTG, the patient reported that his pain reduced to a "4." Repeat ECG and vital signs remained unchanged. Though Case 1 patient's pain abated, since it persisted, he was given a second dose of SL NTG. Within 2 minutes of receiving the second dose of NTG, the patient became bradycardic (30 beats/min) with a stable BP and then became unresponsive, converting to asystolic rhythm. Cardiopulmonary resuscitation (CPR) was initiated, with a successful return of vital signs and baseline cognition following 20 seconds of compressions. Despite success following critical interventions, his HR persisted at 30 beats/min with a narrow regular complex, and normal BP. Because of the persistent bradycardia and preceding asystolic rhythm, he was given $0.5 \mathrm{mg}$ of atropine IV, which increased his HR to 80 beats/min. Cardiology service was consulted, and the patient was admitted following an otherwise stable course. Since the cardiologist did not feel emergent cardiac catheterization was indicated, the patient was observed and subsequently discharged home following an uneventful hospitalization, including a normal stress test.

\section{Case 2}

The patient in Case 2, had chest pain upon arrival at the ED and was administered SL NTG, with notable improvement in chest pain, but not complete resolution. With serial examinations, including a review of pain scale scores, she was given two subsequent doses of SL NTG. Within 1 minute from receiving the third dose of NTG, the patient complained of lightheadedness and nausea, and became pale and diaphoretic. Telemetry revealed bradycardia, which progressed to junctional escape beats, followed by ventricular escape beats, and then asystole, at which point she became unresponsive and pulseless. Cardiopulmonary resuscitation was initiated, with a return of spontaneous circulation within 15 seconds of intervention; she gradually returned to her baseline with observation. Repeat vital signs were: BP, 155/70 mm Hg; HR, 99 beats/min; RR, 20 breaths/min; and she was afebrile. Oxygen saturation was $99 \%$ on 15 liters of oxygen/ min, which was weaned prior to hospital admission. A repeat ECG demonstrated a normal sinus rhythm without evidence of ischemia. Cardiology service was consulted and the patient was admitted for further evaluation, including a 3-day inpatient observation, serial cardiac enzymes, thyroid panel, contrast chest computed tomography scan, echocardiogram, and cardiac stress test. All studies were within normal limits, except for an incidental minor pectus excavatum attributed to the quality CPR. In addition, a nuclear medicine perfusion imaging study was obtained, which revealed no evidence of myocardial ischemia or scar, consistent with the patient's stable course. The patient's symptoms resolved early in her inpatient stay, and she was discharged home with prescriptions for antihypertensive and antihyperlipidemia agents and instructed to follow-up with her primary care physician.

\section{Discussion}

Nitroglycerin is commonly used to treat various symptoms of cardiac origin, namely relief of chest pain due to suspected acute coronary syndromes. ${ }^{2,3}$ The mechanism of action of NTG is predominantly through potent smooth muscle relaxation of the venous and arterial systems, reducing both preload and afterload. ${ }^{2,3}$ This results in reduced myocardial oxygen demand, potentiating the relief of myocardial ischemia.

\section{Contraindications}

Contraindications to NTG include known allergy, pericardial tamponade, restrictive cardiomyopathy, increased intracranial pressure, and concomitant use of phosphodiesterase inhibitors. Moreover, NTG should not be given to treat conditions wherein cardiac 
output is dependent on venous return, as in the setting of inferior myocardial infarction (MI) with right ventricular involvement. Furthermore, there is no evidence in the literature to support the erroneous use of NTG as a diagnostic therapy, with limited sensitivity yields for conclusive cardiac-associated chest pain. ${ }^{8}$

\section{Adverse Effects and Events}

The common side effects of NTG are well documented and include hypotension, tachycardia, flushing, nausea, vomiting, and headache. ${ }^{7,3,6}$ Syncope, bradycardia, and cardiac arrest following the administration of NTG are rare events, as evidenced by the paucity of literature describing these complications. Rather, it appears that these side effects are observed only in the setting of myocardial ischemia or MI., ${ }^{3,-11}$ Fewer cases of ventricular fibrillation, responsive to defibrillation, and asystole also have been observed. ${ }^{9}$ The exact mechanism for bradycardia without hypotension and subsequent asystole following NTG administration remains elusive, though this response is thought to be associated with the Bezold-Jarisch reflex.

\section{Bezold-Jarisch Reflex}

The Bezold-Jarisch reflex is a cardiovascular response consisting of bradycardia and hypotension that is believed to be from stimulation of inhibitory cardiac receptors by stretch, chemical, or pharmacological stimulation. ${ }^{12}$ The earliest cases of Bezold-Jarisch reflex following NTG occurred in the setting of MI and were attributed to ongoing myocardial ischemia. ${ }^{13}$ Recent studies have revealed that coronary stenosis without concurrent ischemia is actually not a sensitizing factor, and that bradycardia and asystole following NTG have occurred in patients without evidence of coronary artery disease. ${ }^{9,14}$ As part of this response, it is theorized that the development of bradycardia is related to vasovagal stimulation, a centrally mediated response to the headache or nausea following NTG administration. ${ }^{10,11,15}$

Despite these observational studies and after thorough review of the available cases, no unifying factors exist to predict with certainty the patient population in which this response is likely to occur. ${ }^{12,16}$ Based on a literature review, it appears that asystole following NTG is self-limited; however, in most cases, bradycardia was treated with atropine without adverse side effects. ${ }^{12,15,16}$

\section{Conclusion}

The two cases presented involved a middle-aged male patient and an elderly female patient, both of whom had several cardiac risk factors but no evidence of acute ischemia or infarction on ECG or laboratory studies. It is well established that NTG can cause hypotension without bradycardia; however, the development of bradycardia without, or even preceding, hypotension is less recognized. Several mechanisms have been postulated but none fully explain this reaction; moreover, no anticipatory risk factors have been consistently observed. Even though the patients in Case 1 and 2 underwent extensive evaluation, no specific etiology of the observed reaction was identified, though neither patient underwent cardiac catheterization to definitively exclude abnormal coronary artery pathology as a precipitating factor.

These cases illustrate the unpredictable adverse reaction to a common medication used for a ubiquitous complaint. The explanation as to the source for this reaction is lacking, the literature has consistently described the transient and self-limiting effect of asystole following NTG. ${ }^{9,12,14,16}$ Bradycardia, though self-limiting, remains responsive to appropriately dosed atropine when NTG-induced., ${ }^{3,12,16}$ The authors wish to stress the 
importance of establishing IV access and being prepared for adverse events whenever administering sublingual nitroglycerin to a patient.

\section{References}

1. Miura T, Nishinaka T, Terada T, Yonezawa K. Vasodilatory effect of nitroglycerin in Japanese subjects with different aldehyde dehydrogenase 2 (ALDH2) genotypes. Chem Biol Interact. 2017;276:40-45. doi:10.1016/j. cbi.2017.03.012.

2. Noonan PK, Williams RL, Benet LZ. Dose dependent pharmacokinetics of nitroglycerin after multiple intravenous infusions in healthy volunteers. J Pharmacokinet Biopharm. 1985;13(2):143-157.

3. Proulx MH, de Montigny L, Ross D, Vacon C, Juste LE, Segal E. Prehospital nitroglycerin in tachycardic chest pain patients: a risk for hypotension or not? Prehosp Emerg Care. 2017;21(1):68-73. doi:10.1080/10903127.2016.119492 9.

4. Huis In 't Veld MA, Cullen L, Mahler SA, Backus BE, Dezman ZDW, Mattu A. The fast and the furious: low-risk chest pain and the rapid rule-out protocol. West J Emerg Med. 2017;18(3):474-478. doi:10.5811/ westjem.2016.12.32676.

5. Pasupathy S, Tavella R, Grover S, et al. Early use of N-acetylcysteine with nitrate therapy in patients undergoing primary percutaneous coronary intervention for ST-segment-elevation myocardial infarction reduces myocardial infarct size (the NACIAM Trial [N-acetylcysteine in Acute Myocardial Infarction]). Circulation. 2017;136(10):894903. doi:10.1161/CIRCULATIONAHA.117.027575.

6. Turan B, Daşlı T, Erkol A, Erden I. Effectiveness of sublingual nitroglycerin before puncture compared with conventional intra-carterial nitroglycerin in transradial procedures: a randomized trial. Cardiovasc Revasc Med. 2015;16(7):391-396. doi:10.1016/j.carrev.2015.07.006.

7. Nagy-Grócz G, Bohár Z, Fejes-Szabó A, et al. Nitroglycerin increases serotonin transporter expression in rat spinal cord but anandamide modulated this effect. J Chem Neuroanat. 2017;85:13-20. doi:10.1016/j.jchemneu.2017.06.002.

8. Steele R, McNaughton T, McConahy M, Lam J. Chest pain in emergency department patients: if the pain is relieved by nitroglycerin, is it more likely to be cardiac chest pain? CJEM. 2006;8(3):164-169.

9. Dettorre K, Brywczynski J, McKinney J, Slovis C. Not the nitro? Patient goes into prehospital V-fib arrest following nitroglycerin. JEMS. 2009;34(5):34,36. doi:10.1016/S0197-2510(09)70124-X.

10. Buckley R, Roberts R. Symptomatic bradycardia following the administration of sublingual nitroglycerin. Am J Emerg Med. 1993;11(3):253-255

11. Takase B, Uehata A, Nishioka T, et al. Different mechanisms of isoproterenol-induced and nitroglycerin-induced syncope during head-up tilt in patients with unexplained syncope: important role of epinephrine in nitroglycerininduced syncope. J Cardiovasc Electrophysiol. 2001:12(7):791-796.

12. Brandes W, Santiago T, Limacher M. Nitroglycerin-induced hypotension, bradycardia, and asystole: report of a case and review of the literature. Clin Cardiol. 1990;13(10):741-744.

13. Ong EA, Canlas C, Smith W. Nitroglycerin-induced asystole. Arch Intern Med. 1985;145(5):954.

14. Shah SP, Waxman S. Two cases of Bezold-Jarisch reflex induced by intra-arterial nitroglycerin in critical left main coronary artery stenosis. Tex Heart Inst J. 2013;40(4):484-486.

15. Mark AL. The Bezold-Jarisch reflex revisited: clinical implications of inhibitory reflexes originating in the heart. J Am Coll Cardiol. 1983;1(1):90-102.

16. Younas F, Janjua M, Badshah A, DeGregorio M, Patel KC, Cotant JF. Transient complete heart block and isolated ventricular asystole with nitroglycerin. J Cardiovasc Med (Hagerstown). 2012;13(8):533-535. doi:10.2459/ JCM.0b013e3283416b8b. 